\title{
Preeclampsia as a Manifestation of New-Onset Systemic Lupus Erythematosus during Pregnancy: A Case-Based Literature Review
}

\author{
Taito Miyamoto, MD ${ }^{1}$ Tatsuji Hoshino, MD, PhD ${ }^{1}$ Nobutaka Hayashi, MD ${ }^{1}$ Ruriko Oyama, MD ${ }^{1}$ \\ Asuka Okunomiya, MD ${ }^{1}$ Sachiko Kitamura, $\mathrm{MD}^{1} \quad$ Noriko Ohtake, $\mathrm{MD}^{1}$ Mami Suga, MD ${ }^{1}$ \\ Kazunao Miyamoto, MD ${ }^{1}$ Aki Takaoka, MD ${ }^{1}$ Takuya Aoki, MD ${ }^{1}$ Yuko Imamura, MD ${ }^{1}$ \\ Seiji Nagano, MD² Masato Kita, MD, $\mathrm{PhD}^{1}$
}

${ }^{1}$ Department of Obstetrics and Gynecology, Kobe City Medical Center General Hospital, Kobe, Japan

2 Department of Hematology and Clinical Immunology, Kobe City Medical Center General Hospital, Kobe, Japan
Address for correspondence Taito Miyamoto, MD, Department of Obstetrics and Gynecology, Kobe City Medical Center General Hospital, 2-1-1 Minatojima-Minamimachi, Chuo-ku, Kobe, Hyogo 6500047, Japan (e-mail: taitom33@yahoo.co.jp).

Am J Perinatol Rep 2016;6:e62-e67.

\begin{abstract}
Keywords

- systemic lupus erythematosus

- onset during pregnancy

- seizure

- preeclampsia

- thrombocytopenia

Introduction New-onset systemic lupus erythematosus (SLE) during pregnancy is rare and difficult to diagnose, especially in cases that manifest as preeclampsia. We report a patient with new-onset SLE that manifested as preeclampsia during pregnancy and provide a review of the literature to identify factors for a rapid diagnosis.

Case A 32-year-old primigravid Japanese woman was diagnosed with severe preeclampsia and underwent emergent cesarean section at 29 weeks of gestation. Her hypertension and renal disorder gradually improved after the operation, but her thrombocytopenia and anemia worsened. SLE was diagnosed on postoperative day 5 by a comprehensive autoimmune workup. She was discharged on postoperative day 34 with remission.

Conclusion Our case and previous reports suggest that distinguishing underlying SLE from preeclampsia in the third trimester is particularly difficult. Helpful factors for diagnosis of suspected SLE in these cases were persistence of symptoms and new atypical symptoms for preeclampsia revealed after delivery (e.g., fever, renal disorder, and thrombocytopenia).
\end{abstract}

Systemic lupus erythematosus (SLE) is a systemic autoimmune disease that primarily affects women in their reproductive years. Lupus occurred in 13,555 of 16.7 million pregnancies in a recent study in the United States. ${ }^{1}$ Most cases of SLE in pregnancy are diagnosed before conception, and new-onset SLE during pregnancy and in the postpartum period is rare, occurring in 0.0 to $13.5 \%$ of pregnancies with SLE according to recent reports. ${ }^{2-6}$ Preeclampsia is much more common than new-onset SLE during pregnancy. In the United States, preeclampsia occurs in 5 to $8 \%$ of the general population and in 13 to $35 \%$ of pregnant women with SLE. ${ }^{7}$ The signs and symptoms of lupus flare-ups may mimic those of preeclampsia, and distinguishing new-onset SLE during pregnancy from preeclampsia is sometimes difficult. The clinical picture is particularly complicated if the initial manifestation is preeclampsia.

We present here a challenging case of new-onset SLE that manifested as preeclampsia during pregnancy. Such a situation could result in catastrophic illness with dire maternal and fetal outcomes if diagnosis and management are delayed. received

August 6, 2015 accepted after revision September 11, 2015 published online November 3, 2015
DOI http://dx.doi.org/ 10.1055/s-0035-1566245. ISSN 2157-6998.
Copyright $\odot 2016$ by Thieme Medical Publishers, Inc., 333 Seventh Avenue, New York, NY 10001, USA. Tel: +1(212) 584-4662.
License terms

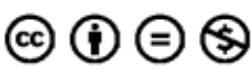


We also reviewed the literature on new-onset SLE that manifested as preeclampsia during pregnancy and in the postpartum period, and discuss facilitation of rapid diagnosis.

\section{Case Study}

A 32-year-old primigravid Japanese woman with no remarkable medical history had become pregnant by in vitro fertilization and egg transfer. She underwent a prenatal checkup by a local doctor. Laboratory testing during the first trimester revealed anemia (hemoglobin level of $9.0 \mathrm{~g} / \mathrm{dL}$ ), thrombocytopenia (platelet count of $7.0 \times 10 / \mu \mathrm{L}$ ), and proteinuria $(2+)$, but no further assessment was performed. When she was referred to a clinic near her parents' home at 29 weeks of gestation, leg edema, hypertension, intrauterine growth reduction ( -2.3 standard deviation), and a high umbilical artery resistance index were observed. She was transferred to a city hospital and underwent further examination. On the same day, she was diagnosed with severe preeclampsia and a nonreassuring fetal status based on severe hypertension $(160 / 100 \mathrm{~mm} \mathrm{Hg})$, thrombocytopenia $(2.9 \times 10 / \mu \mathrm{L})$, renal disorder (serum creatinine level of 1.25 $\mathrm{mg} / \mathrm{dL}$ ), and loss of variability on cardiotocography. She was transferred to our hospital immediately. An emergent cesarean section was performed under general anesthesia with preoperative transfusion of 10 units of concentrated platelets. A 1,048-g female infant was born with Apgar scores of 3 and 9 at 1 and 5 minutes, respectively. Although no major intraoperative problems occurred, awakening of the patient was delayed despite the cessation of anesthetic administration. She suddenly showed general convulsions in the operating room. An emergency computed tomographic examination of the head revealed no hemorrhage or infarction. We suspected eclampsia and began intravenous administration of magnesium sulfate, nicardipine hydrochloride, and unfractionated heparin. Magnetic resonance imaging the next day showed no occipital lobe lesions characteristic of posterior reversible encephalopathy syndrome. The general convulsions did not recur. Although her consciousness, hypertension, and serum creatinine levels had improved, her thrombocytopenia and anemia persisted. Therefore, frequent platelet transfusions were required (-Fig. 1). On postoperative day (POD) 3, we suspected an underlying autoimmune disorder, and a complete autoimmune workup was initiated. On POD4, we started intravenous prednisolone administration before diagnosis. Laboratory findings the following day showed positivity for antinuclear antibody, anti-ds DNA antibody, anti-Sm antibody, and anti-RNP antibody $(72.5,21,170$, and $109 \mathrm{IU} / \mathrm{mL}$, respectively), and low C3 and C4 complement levels (42 and 8 $\mathrm{mg} / \mathrm{dL}$, respectively). Antiphospholipid antibodies, anti-Ro, and anti-La antibodies, antineutrophil cytoplasmic antibodies, antiplatelet factor 4/heparin antibodies, broken erythrocytes, and a disintegrin-like and metalloproteinase with thrombospondin type 1 motif 13 were negative. Plateletassociated IgG was almost normal ( $43.8 \mathrm{ng} / 10^{7}$ cells). Based on these findings, she was diagnosed with SLE on POD5. Several days later, her platelet count and hemoglobin level increased to the reference ranges. Her proteinuria improved from $4 \mathrm{~g} / \mathrm{d}$ on POD4 to $1 \mathrm{~g} / \mathrm{d}$ on POD18, and her blood pressure normalized on POD7. The patient was discharged on POD34 with normal blood pressure, normal laboratory data, and no recurrence of seizures. Her SLE has been well controlled with oral prednisone. Evaluation of her newborn showed no evidence of neonatal lupus.

\section{Discussion}

SLE predominantly affects young women of child-bearing age. There have been numerous studies of pregnant women diagnosed with SLE before conception, and the course of pregnancy with SLE has dramatically improved in recent decades. The average probability of a lupus flare during pregnancy and the postpartum period has decreased from $60.0 \%$ in the presteroid era to $23.3 \%$ at the beginning of the 21st century. ${ }^{6}$ There is no consensus on whether the incidence of lupus flare increases during pregnancy and the postpartum period or in which trimester it is most associated with a risk of lupus flare. However, SLE activity during the 6 months preceding conception and preexisting renal disease are known as potential risk factors for flares during pregnancy. There is a greater prevalence of preeclampsia, fetal loss, intrauterine growth retardation, and preterm birth among women with active lupus during pregnancy. ${ }^{6}$

However, there are few reports on SLE during pregnancy and in the postpartum period. A literature search of MEDLINE and Japana Centra Revuo Medicina for reports published during the past 20 years showed only 13 case reports involving 16 patients, ${ }^{8-20}$ one comparative study on newonset SLE during pregnancy and the postpartum period, ${ }^{21}$ and two studies that presented limited information. ${ }^{2,3}$

Among all 17 reported patients (16 previous reports plus the present case) (-Table $\mathbf{1}$ ), the mean maternal age was 28 years (range, 20-34 years). Excluding two cases in which such information was not provided; two cases involved abortion, nine involved preterm delivery, and only four involved term delivery. No cases of stillbirth or neonatal death were reported. Preeclampsia/eclampsia was the most common complication and was found in 11 (65\%) cases. Hemophagocytic syndrome, acute fatty liver, HELLP (hemolysis, elevated liver enzymes, and low platelet count) syndrome, and myocardial infarction were the other severe complications, but no maternal death was reported. The numbers of patients who manifested specific diagnostic criteria of SLE included the following: malar rash in 7 (41\%); discoid rash in 2 (12\%); photosensitivity in $0(0 \%)$; oral ulcers in 3 (18\%); arthritis in 4 (24\%); serositis in $4(24 \%)$; renal disorder in 11 (65\%); neurological disorders in $3(18 \%)$; leukopenia in 7 (41\%); anemia in 6 (35\%); thrombocytopenia in 6 (35\%); anti-ds DNA in 14 (82\%); and antinuclear antibody in $17(100 \%)$. Renal disorders appeared to be more common at the onset of SLE in pregnant patients than in nonpregnant patients.

Zhao et $\mathrm{al}^{21}$ described 48 patients with new-onset SLE during pregnancy. These patients' clinical characteristics and serological parameters were retrospectively compared with those of age-matched patients with new-onset SLE who were 


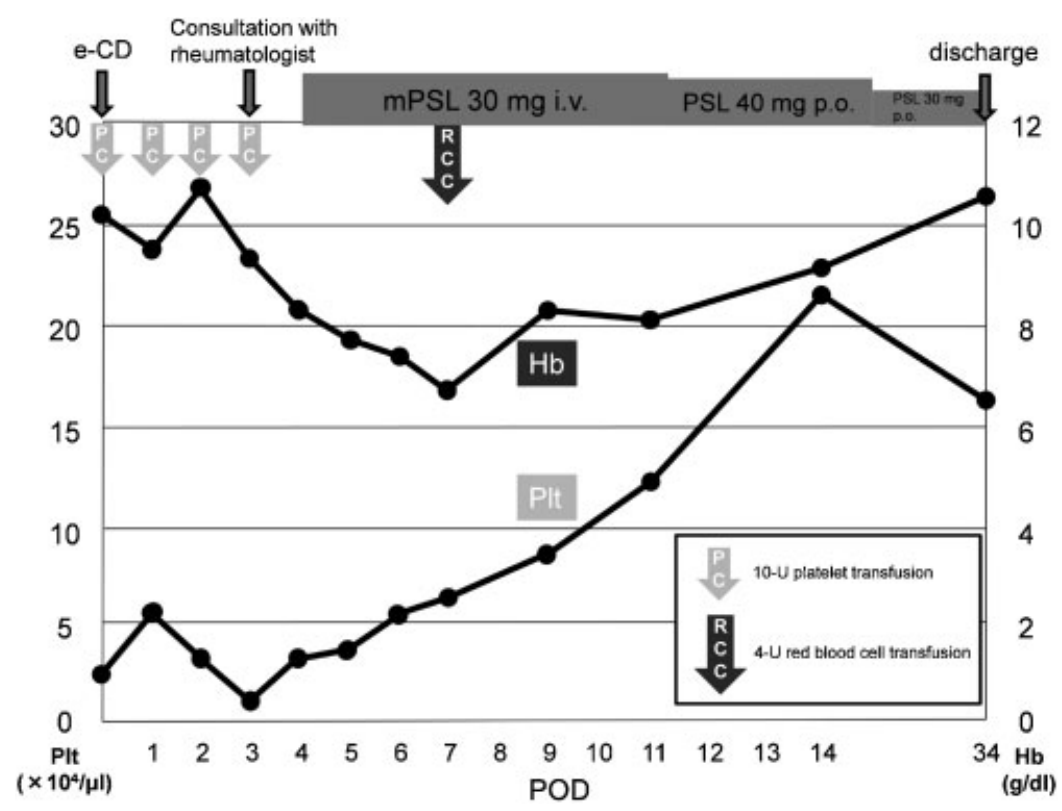

Fig. 1 Changes in platelet and hemoglobin levels. e-CD, emergent cesarean delivery; mPSL, methylprednisolone; PC, platelet concentrate; POD, postoperative day; PSL, prednisolone; RCC, red cell concentrate.

not pregnant within 12 months before the diagnosis. Lupus nephritis (68.8\%) and severe thrombocytopenia (25.0\%) were more commonly observed and arthritis/arthralgia (8.3\%), alopecia (4.2\%), and ulceration (2.1\%) were less commonly found in patients with new-onset SLE during pregnancy than in patients who were not pregnant. Only two (4.2\%) patients had the complication of preeclampsia. However, detailed data on each patient are not available. Chakravarty et $\mathrm{al}^{2}$ and Chandran et $\mathrm{al}^{3}$ reported that few cases of new-onset SLE during pregnancy showed poor maternal and neonatal outcomes in their studies. However, detailed information on each patient is not available.

Of the 11 patients who developed preeclampsia/eclampsia in the above-described case reports, 3 developed preeclampsia after the diagnosis of SLE and 8 had SLE that manifested as preeclampsia that had not been initially diagnosed. In these eight patients, preeclampsia mostly occurred in the third trimester, with the exception of one patient who developed preeclampsia immediately after delivery. Of these seven patients, three developed preeclampsia as the first manifestation of SLE. The remaining four patients (including our patient) developed preeclampsia with preceding symptoms of SLE, such as leg edema, proteinuria, mild anemia, and thrombocytopenia, which are also commonly found in patients with preeclampsia, or even in patients with a normal pregnancy. No patients developed other typical symptoms of SLE (e.g., malar rash or arthritis) as the first manifestation of SLE in the third trimester or preceding preeclampsia before the diagnosis of SLE. These findings suggest that distinguishing underlying SLE from preeclampsia is difficult, especially in the third trimester, because other typical symptoms of SLE are not initially observed. Moreover, preeclampsia is common not only in patients with SLE, but also in healthy women in their third trimester of pregnancy. In six of the seven above- mentioned patients, the pregnancy was terminated to treat the preeclampsia before the diagnosis of SLE, and all patients had a preterm delivery.

The diagnosis of SLE tended to be delayed ( 5 days to $>12$ months after delivery) in the eight cases that manifested as preeclampsia because termination of pregnancy was believed to be sufficient to improve the condition, and an immunological examination was not initially performed. An autoimmune disorder was finally suspected when the condition worsened, even after parturition. Important factors for identifying underlying SLE were the persistence or new onset of the following abnormal physical or laboratory findings: malar rash, fever, purpuric macules on the fingers, acute heart failure, serositis, renal disorder, cytopenia, and recurrence of seizures.

In our case, the anemia, thrombocytopenia, and proteinuria that were incidentally found in the first trimester led us to the correct diagnosis. The persistence of thrombocytopenia and worsening anemia helped us to suspect an underlying autoimmune disease. When the platelet count does not reach its nadir for 48 to 72 hours, preeclampsia syndrome may be incorrectly attributed to a thrombotic microangiopathy. Thrombotic thrombocytopenic purpura, hemolytic uremic syndrome, HELLP syndrome, acute fatty liver, or catastrophic antiphospholipid syndrome, all of which were ruled out by biochemical and immunological examinations, were considered to be the differential diagnoses.

In conclusion, new-onset SLE during pregnancy is rare and difficult to quickly diagnose, especially in cases that manifest as preeclampsia in the third trimester. New-onset SLE should be suspected and an autoimmune workup should be performed as soon as possible when symptoms of preeclampsia persist or new atypical symptoms of preeclampsia develop after delivery. 
Preeclampsia as a Manifestation of New-Onset SLE Miyamoto et al. e65

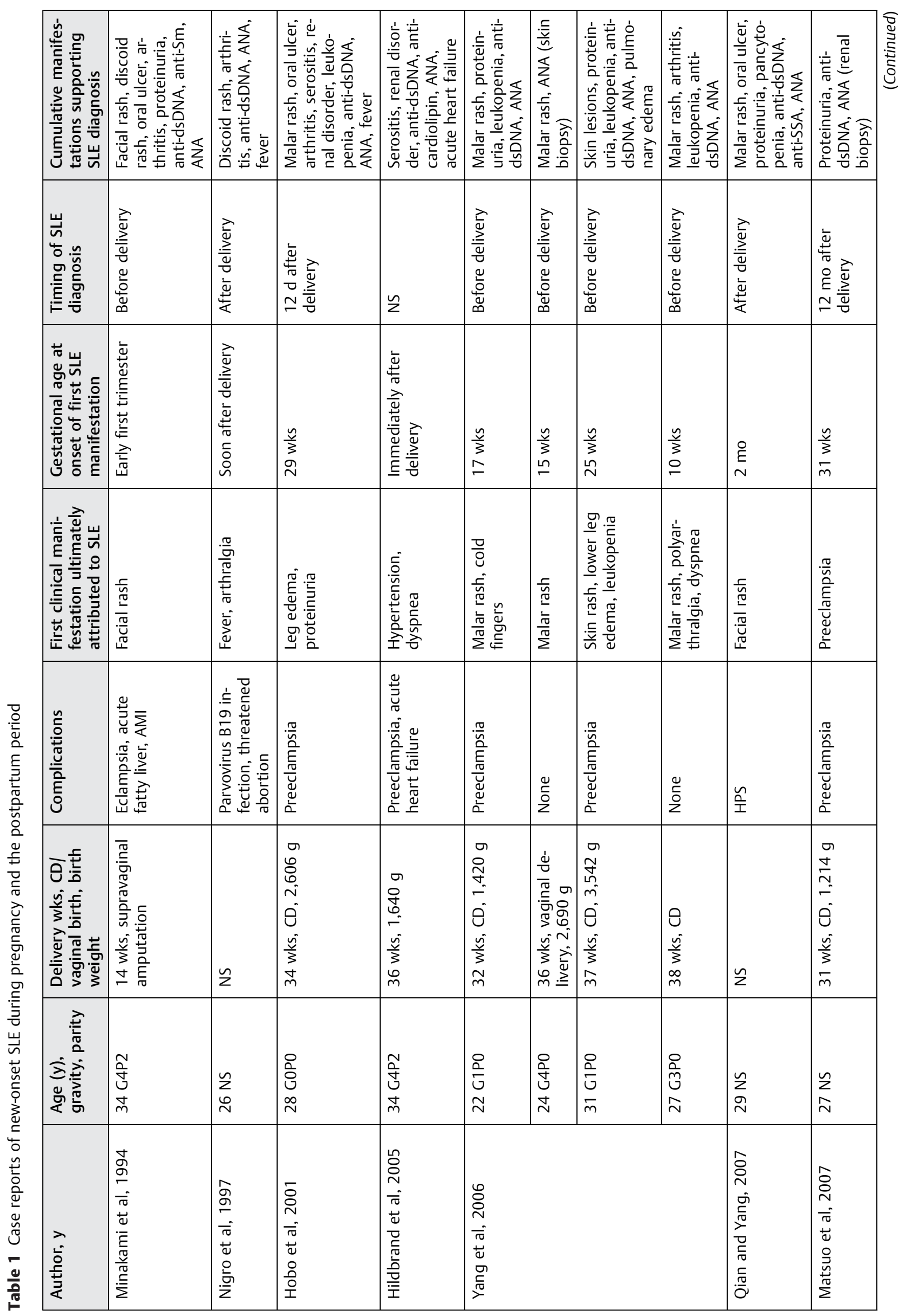




\begin{tabular}{|c|c|c|c|c|c|c|c|}
\hline 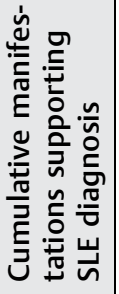 & 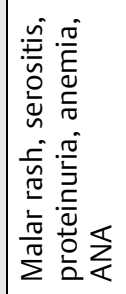 & 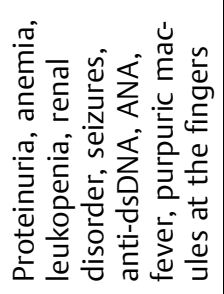 & 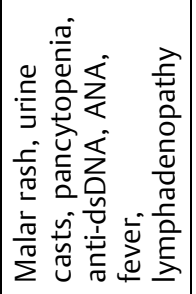 & 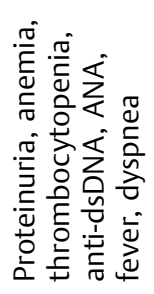 & 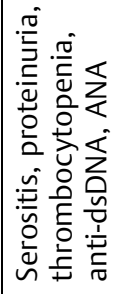 & 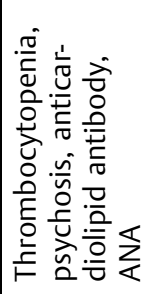 & 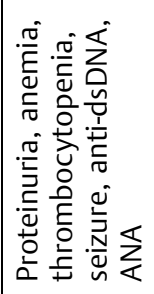 \\
\hline 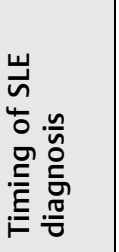 & 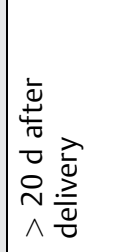 & 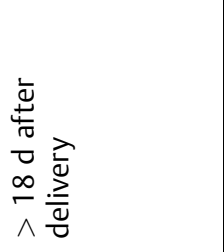 & 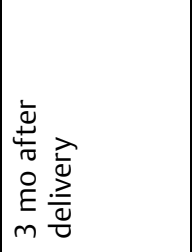 & 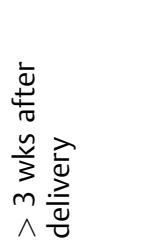 & 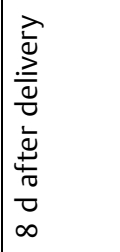 & 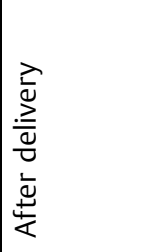 & 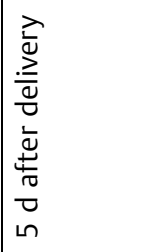 \\
\hline 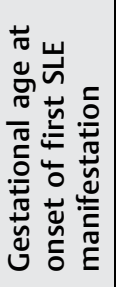 & 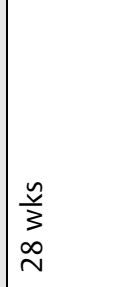 & $\begin{array}{l}\frac{\tilde{y}}{3} \\
9 \\
m\end{array}$ & 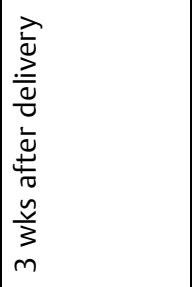 & $\begin{array}{l}\frac{\tilde{y}}{3} \\
\frac{m}{m}\end{array}$ & $\begin{array}{l}\tilde{n} \\
\stackrel{\tilde{z}}{N} \\
\stackrel{\nu}{2}\end{array}$ & 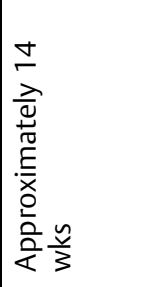 & $\begin{array}{l}\tilde{y} \\
\underline{\underline{s}} \\
\underline{\underline{0}}\end{array}$ \\
\hline 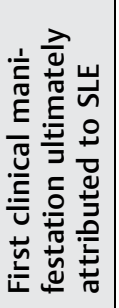 & 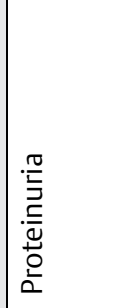 & 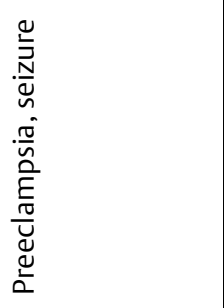 & 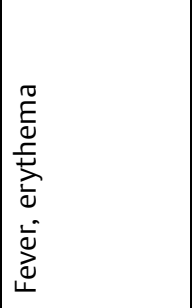 & 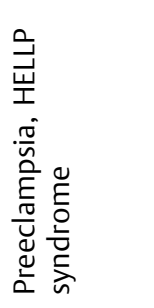 & 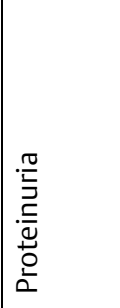 & 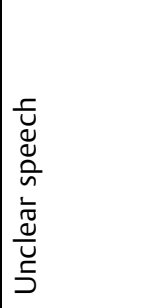 & 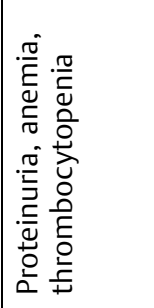 \\
\hline 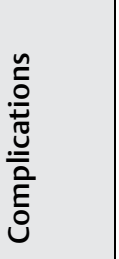 & 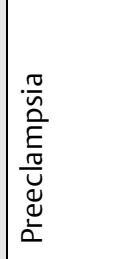 & 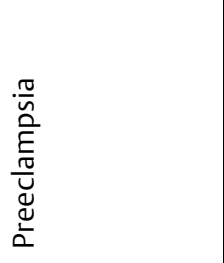 & $\stackrel{n}{\underline{I}}$ & 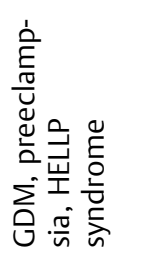 & 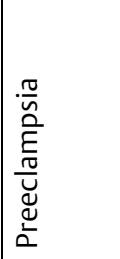 & 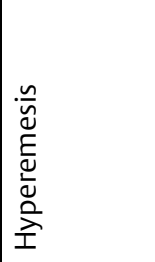 & 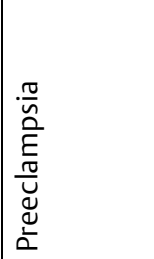 \\
\hline 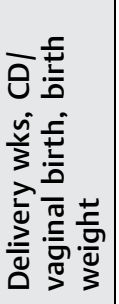 & 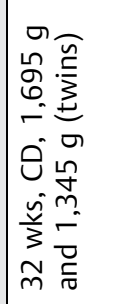 & $\begin{array}{l}0 \\
\dot{v} \\
\dot{v} \\
\text { s. } \\
m\end{array}$ & 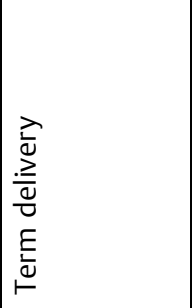 & $\begin{array}{l}0 \\
8 \\
0 \\
\vdots \\
\vdots \\
0 \\
\dot{v} \\
\frac{5}{3} \\
\bar{m}\end{array}$ & 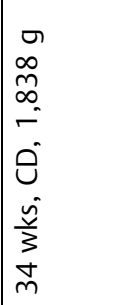 & 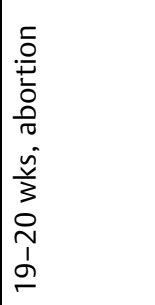 & 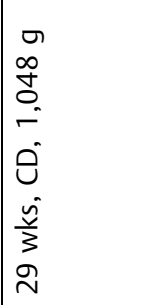 \\
\hline 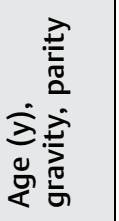 & $\begin{array}{l}\bar{\Sigma} \\
\bar{v} \\
\grave{N}\end{array}$ & $\begin{array}{l}\stackrel{\circ}{0} \\
\text { Oे } \\
\stackrel{2}{N}\end{array}$ & $\begin{array}{l}\circ \\
m \\
m\end{array}$ & $\begin{array}{l}\circ \\
0 \\
0 \\
\stackrel{0}{0} \\
\text { N }\end{array}$ & $\begin{array}{l}\stackrel{0}{0} \\
0 \\
\text { D } \\
\stackrel{N}{N}\end{array}$ & $\begin{array}{l}\underset{\infty}{\sim} \\
\underset{\sim}{N}\end{array}$ & 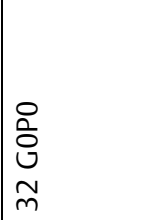 \\
\hline 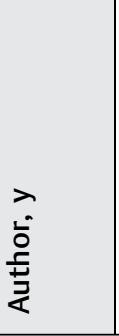 & 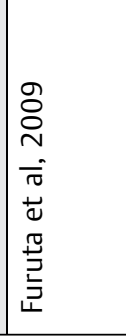 & 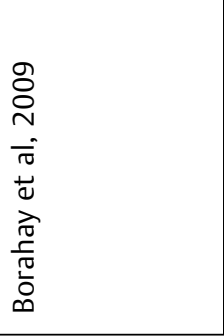 & 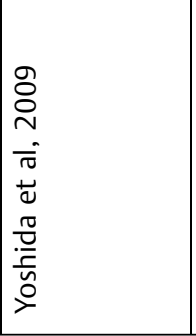 & 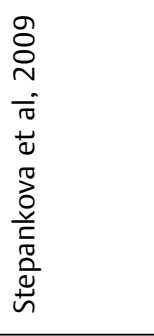 & 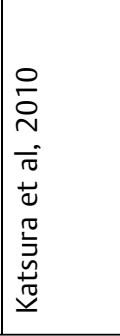 & 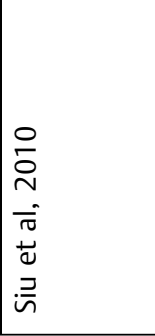 & 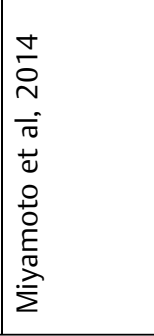 \\
\hline
\end{tabular}




\section{References}

1 Clowse ME, Jamison M, Myers E, James AH. A national study of the complications of lupus in pregnancy. Am J Obstet Gynecol 2008; 199(2):127.e1-127.e6

2 Chakravarty EF, Colón I, Langen ES, et al. Factors that predict prematurity and preeclampsia in pregnancies that are complicated by systemic lupus erythematosus. Am J Obstet Gynecol 2005; 192(6):1897-1904

3 Chandran V, Aggarwal A, Misra R. Active disease during pregnancy is associated with poor foetal outcome in Indian patients with systemic lupus erythematosus. Rheumatol Int 2005;26(2): 152-156

4 Clowse ME, Magder LS, Witter F, Petri M. The impact of increased lupus activity on obstetric outcomes. Arthritis Rheum 2005;52(2): 514-521

5 Cortés-Hernández J, Ordi-Ros J, Paredes F, Casellas M, Castillo F, Vilardell-Tarres M. Clinical predictors of fetal and maternal outcome in systemic lupus erythematosus: a prospective study of 103 pregnancies. Rheumatology (Oxford) 2002;41(6): 643-650

6 Karpouzas GA, Kitridou RC. The mother in systemic lupus erythematosus. In: Wallace DJ, Hahn BH eds. Dubois' Lupus Erythematosus. Philadelphia, PA: Lippincott Williams \& Wilkins; 2007: 992-1038

7 Stojan G, Baer AN. Flares of systemic lupus erythematosus during pregnancy and the puerperium: prevention, diagnosis and management. Expert Rev Clin Immunol 2012;8(5):439-453

8 Minakami H, Idei S, Koike T, Tamada T, Yasuda Y, Hirota N. Active lupus and preeclampsia: a life threatening combination. J Rheumatol 1994;21(8):1562-1563

9 Nigro G, Piazze J, Taliani G, Mazzocco M, Cassinotti P, Cosmi EV. Postpartum lupus erythematosus associated with parvovirus B19 infection. J Rheumatol 1997;24(5):968-970

10 Hobo R, Hayashi M, Saitou M, Hayashi N, Takeda S, Kinoshita K. A case of systemic lupus erythematosus with superimposed severe preeclampsia diagnosed in the postpartum [in Japanese]. Nihon Sanka Fujinnka Gakkai Kanto Rengo Chiho Bukai Kaiho 2001; 38:385-388
11 Hildbrand P, Eigenmann C, Gugger M, Marti HP, Hullin R. Preeclampsia with acute heart failure postpartum as primary manifestation of systemic lupus erythematosus. Eur J Heart Fail 2005; 7(6):1057-1058

12 Yang MJ, Cheng MH, Lin HY. Onset of systemic lupus erythematosus during pregnancy. J Chin Med Assoc 2006;69(3): 130-133

13 Qian J, Yang CD. Hemophagocytic syndrome as one of main manifestations in untreated systemic lupus erythematosus: two case reports and literature review. Clin Rheumatol 2007;26(5): 807-810

14 Matsuo S, Uchida E, Shimajiri S, Nishihara G. A case of a pregnant woman developing systemic lupus erythematosus during pregnancy. Clin Nephrol 2007;67(5):331-332

15 Furuta Y, Sirogane T, Wakui Y, Yamada H, Sakai K. Sotai ninnsinn kannrityuu ni hassyou sita zennshinnsei eritematodesu. Hokkaido Sanka Fujinnka Gakkai Kaishi 2009;52:28-30

16 Borahay MA, Kelly BC, Harirah HM. Systemic lupus erythematosus presenting with leukocytoclastic vasculitis and seizure during pregnancy. Am J Perinatol 2009;26(6):431-435

17 Yoshida S, Takeuchi T, Itami Y, et al. Hemophagocytic syndrome as primary manifestation in a patient with systemic lupus erythematosus after parturition [in Japanese]. Nihon Rinsho Meneki Gakkai Kaishi 2009;32(1):66-70 Japanese

18 Stepanková J, Bürgelova M, Honsova E, Tesar V, Vrbska J, Viklicky O. Preeclampsia, dilated cardiomyopathy and renal failure as the first manifestation of systemic lupus erythematosus: a case report. Clin Rheumatol 2009;28(3):343-345

19 Katsura D, OnoT, Takahashi A, et al. Ninshintyuu kessyoubann gensho wo kitashi, bunnbenngo ni jinn shogai ga shinko shi SLE to shindann sareta ichirei. Shigaken Sanka Fujinnka Zasshi 2010;2:29-31

20 Siu BW, Chow HM, Kwok SS, Li OL, Koo ML, Poon PW. Systemic lupus erythematosus as a cause of first-episode psychosis in the second trimester of pregnancy. East Asian Arch Psychiatry 2010; 20(3): $145-150$

21 Zhao C, Zhao J, Huang Y, et al. New-onset systemic lupus erythematosus during pregnancy. Clin Rheumatol 2013;32(6): 815-822 